EUROPEAN ORGANIZATION FOR NUCLEAR RESEARCH

Laboratory for Particle Physics

Divisional Report

CERN LHC/2001-5

(ECR)

\title{
THE HELIUM CRYOGENIC PLANT FOR THE CMS SUPERCONDUCTING MAGNET
}

\author{
G. Perinic ${ }^{1}$, A. Caillaud ${ }^{2}$, F. Dagut ${ }^{2}$, P. Dauguet ${ }^{2}$ and P. Hirel $^{2}$
}

\begin{abstract}
A new helium refrigeration plant with a cooling capacity of $800 \mathrm{~W}$ at $4.45 \mathrm{~K}, 4500 \mathrm{~W}$ between $60 \mathrm{~K}$ and $80 \mathrm{~K}$, and $4 \mathrm{~g} / \mathrm{s}$ liquefaction simultaneously has been designed and is presently being constructed by Air Liquide for CERN. The refrigeration plant will provide the cooling power for the cool down and the operation of the CMS (Compact Muon Solenoid) superconducting coil whose cold mass weighs $225 \mathrm{t}$. The refrigeration plant will at first be installed in a surface building for the tests of the superconducting magnet. On completion of the tests the cold box will be moved to its final underground position next to the CMS experimental cavern.

This paper presents the process design, describes the main components and explains their selection.
\end{abstract}

1 CERN, LHC-Division

2 Air Liquide, Advanced Technology Division, Sassenage, France

Presented at the 2001 Cryogenic Engineering Conference and International Cryogenic Materials Conference CEC/ICMC 2001

16-20 July 2001, Madison, Wisconsin, USA

Administrative Secretariat LHC Division CERN

CH - 1211 Geneva 23
Geneva, Switzerland

29 October 2001 


\title{
THE HELIUM CRYOGENIC PLANT FOR THE CMS SUPERCONDUCTING MAGNET
}

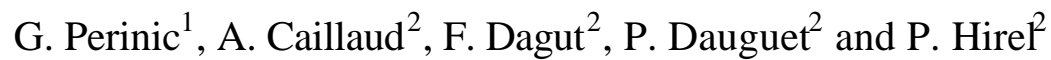 \\ ${ }^{1}$ CERN, LHC-Division \\ CH-1211 Genève 23, Switzerland \\ ${ }^{2}$ Air Liquide, Advanced Technology Division \\ F-38360 Sassenage, France
}

\begin{abstract}
A new helium refrigeration plant with a cooling capacity of $800 \mathrm{~W}$ at $4.45 \mathrm{~K}, 4500 \mathrm{~W}$ between $60 \mathrm{~K}$ and $80 \mathrm{~K}$, and $4 \mathrm{~g} / \mathrm{s}$ liquefaction simultaneously has been designed and is presently being constructed by Air Liquide for CERN. The refrigeration plant will provide the cooling power for the cool down and the operation of the CMS (Compact Muon Solenoid) superconducting coil whose cold mass weighs $225 \mathrm{t}$. The refrigeration plant will at first be installed in a surface building for the tests of the superconducting magnet. On completion of the tests the cold box will be moved to its final underground position next to the CMS experimental cavern.

This paper presents the process design, describes the main components and explains their selection.
\end{abstract}

\section{INTRODUCTION}

The CMS detector of the Large Hadron Collider (LHC) is described elsewhere [2]. Its design has been optimised for muon detection by the utilisation of a compact magnet that can generate a very strong magnetic field. The chosen magnet is a $12.5 \mathrm{~m}$ long superconducting solenoid with an internal bore of $5.9 \mathrm{~m}$, which will generate a magnetic field of $4 \mathrm{~T}$. The $225 \mathrm{t}$ cold mass of the magnet will be cooled by a thermosiphon cooling system.

The design task for the cryogenic system has been split up into two parts for construction - the internal and the external cryogenic sub-systems. The internal cryogenic system comprises the thermosiphon cooling circuits, the thermal shields of the cold mass, the phase separator which will be located on top of the detector, and finally the 


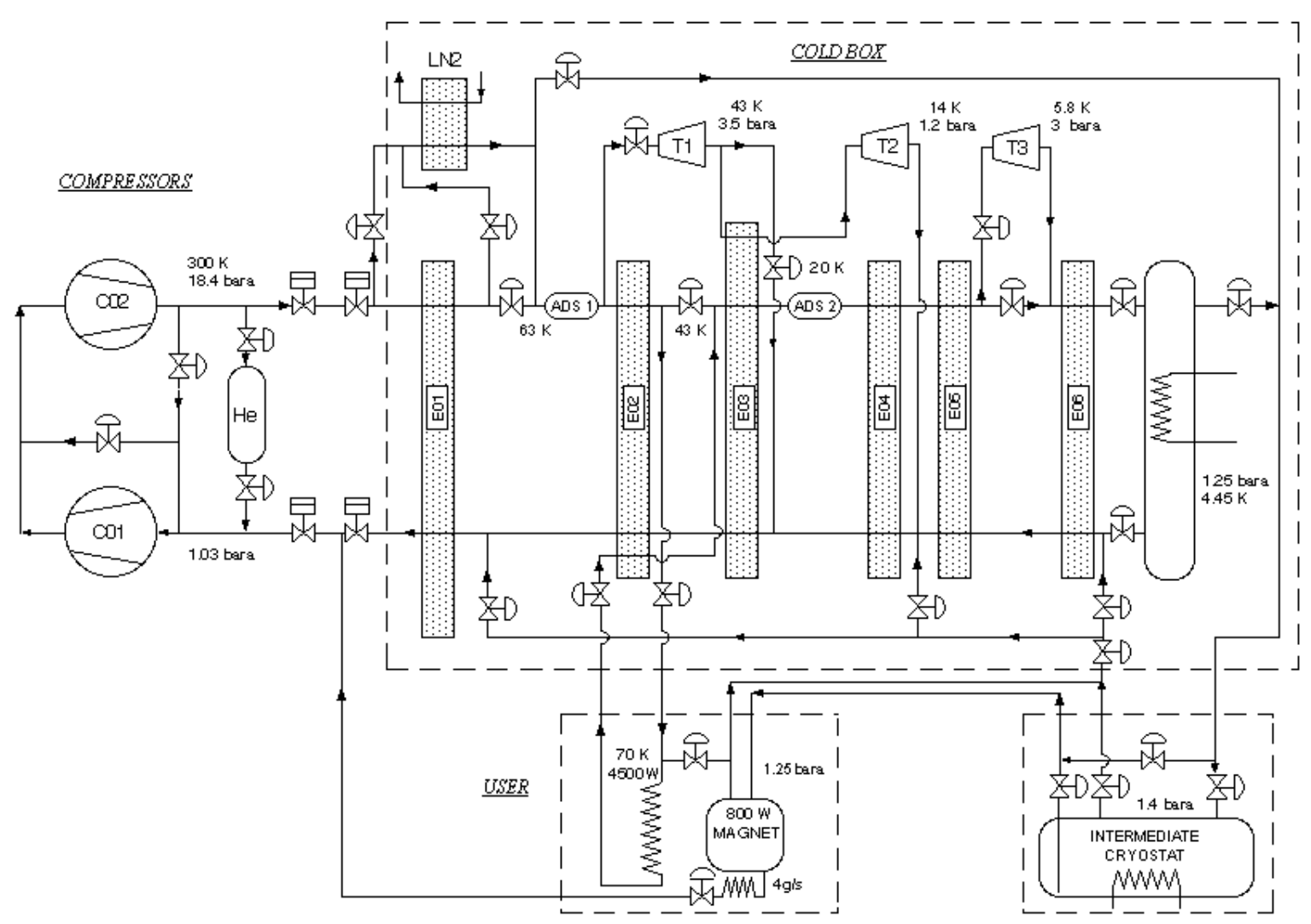

FIGURE 1. Simplified process flow diagram of the CMS refrigeration system.

cryogenic chimney, which connects the cold mass circuits with the phase separator. The design and construction of the internal cryogenic system is the responsibility of the DAPNIA group within CEA at Saclay.

The external cryogenic system consists of the helium refrigeration plant, the transfer line system and an intermediate buffer cryostat with 60001 of liquid helium. For this part, CERN has placed a contract with Air Liquide for design, construction and commissioning, excluding only the helium storage and the control system, which are supplied by other CERN contracts.

\section{PROCESS DESCRIPTION AND DESIGN PERFORMANCE DATA}

The CMS refrigeration system has been designed in view of two main operation modes: cool-down from $300 \mathrm{~K}$ to $4.45 \mathrm{~K}$ and operation at $4.45 \mathrm{~K}$. The refrigeration cycle is based on a modified Claude cycle. Its process flow diagram is shown in FIGURE 1.

During cool-down the refrigerator will supply up to $30 \mathrm{~kW}$ refrigeration power by making use of a liquid nitrogen precooler. This will allow cool down of the $225 \mathrm{t}$ cold mass from $300 \mathrm{~K}$ to $100 \mathrm{~K}$ within 13 days. Further cool down from $100 \mathrm{~K}$ to $4.5 \mathrm{~K}$ will be accomplished with the expansion turbines in 12 days.

During normal operation the refrigerator shall supply $4.5 \mathrm{~kW}$ of refrigeration power between $60 \mathrm{~K}$ and $80 \mathrm{~K}$ for shield cooling, $800 \mathrm{~W}$ refrigeration power at $4.45 \mathrm{~K}$ for the magnet operation, and $4 \mathrm{~g} / \mathrm{s}$ liquefaction power for the current leads cooling in parallel. FIGURE 2 shows the refrigerator cycle in normal operation on a TS-diagram. 


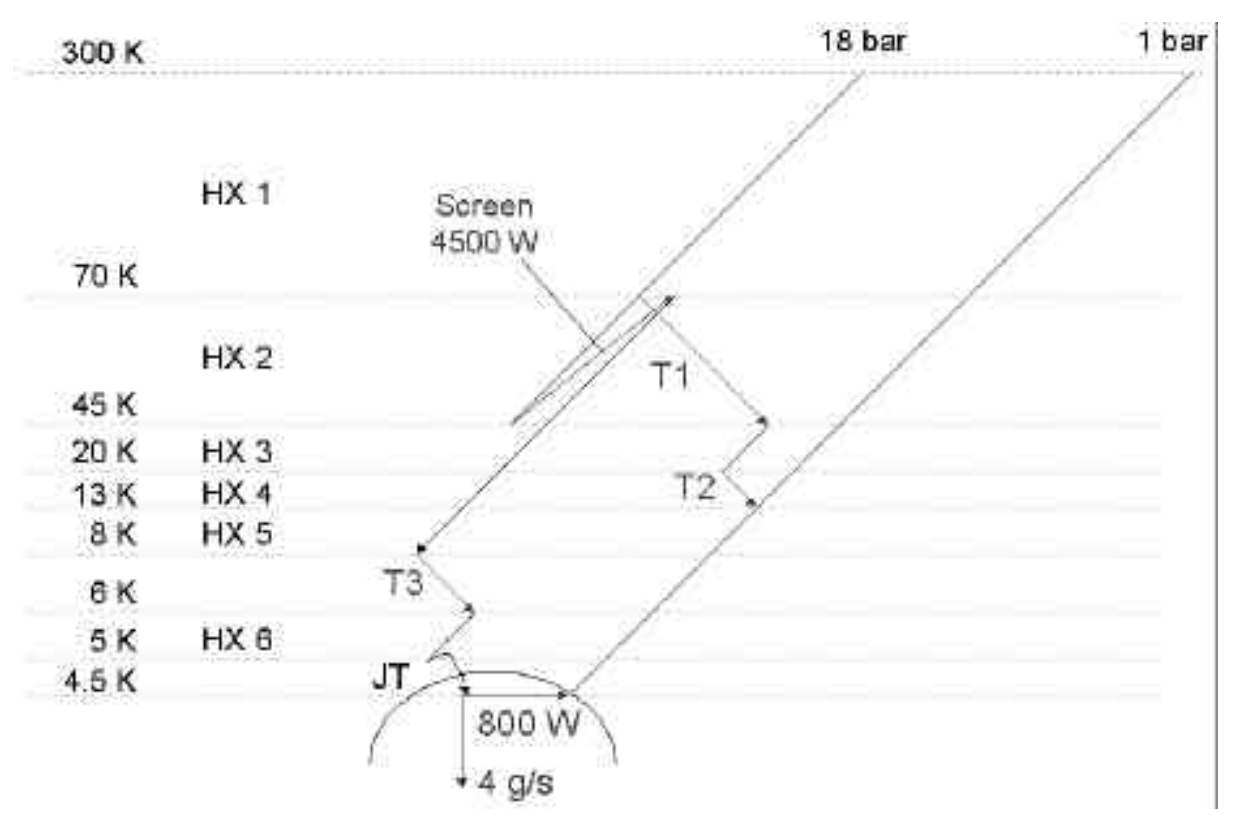

FIGURE 2. Normal operation mode of the CMS refrigerator shown in the TS-diagram.

The refrigerator is divided into three main subsystems: the compressor station, the cold box, and the intermediate cryostat.

\section{DESCRIPTION OF MAIN COMPONENTS}

\section{Compressor Station}

The compression of the helium from 1.03 bar to 18 bar is achieved by the means of two oil-lubricated screw compressors in series - a WLV 321 and a WLV 204 by Howden ${ }^{\text {TM }}$ - providing a mass flow of $207 \mathrm{~g} / \mathrm{s}$. Each compressor is equipped with a directly coupled motor. The second stage compressor serves in case of cryoplant failure as a recovery compressor and allows the storage of $40 \mathrm{~g} / \mathrm{s}$ of helium in the helium buffers.

Screw compressors have been chosen due to their high efficiency, their low temperature isothermal operating condition, and their low vibration level. Furthermore screw compressors are highly reliable and they offer long periods in between maintenance as has been proven in industry where they are applied for industrial refrigeration and air compression, applications that demand high reliabilities - and likewise in many years of operation at CERN.

For the primary oil separation, the oil circulation circuits as well as the helium and oil coolers for the two compressor units are grouped together on one skid. The primary oil separation for the first stage consists of a horizontally installed cylindrical vessel that also serves as the oil reservoir. The primary oil separation for the second stage is a vertical design and is composed of an oil reservoir and of six internal oil coalescing filter cartridges in parallel.

The final oil separation is composed of three coalescing filters and one oil adsorber containing active charcoal all in series. At the outlet of this final oil removal system, the high pressure helium shall contain less than $10 \mathrm{ppb}$ of oil by weight.

The cooling circuits for helium and oil are connected in series for each compression stage. The helium will be cooled from $84^{\circ} \mathrm{C}$ and $81^{\circ} \mathrm{C}$ to $27^{\circ} \mathrm{C}$ and $30^{\circ} \mathrm{C}$ respectively, for 


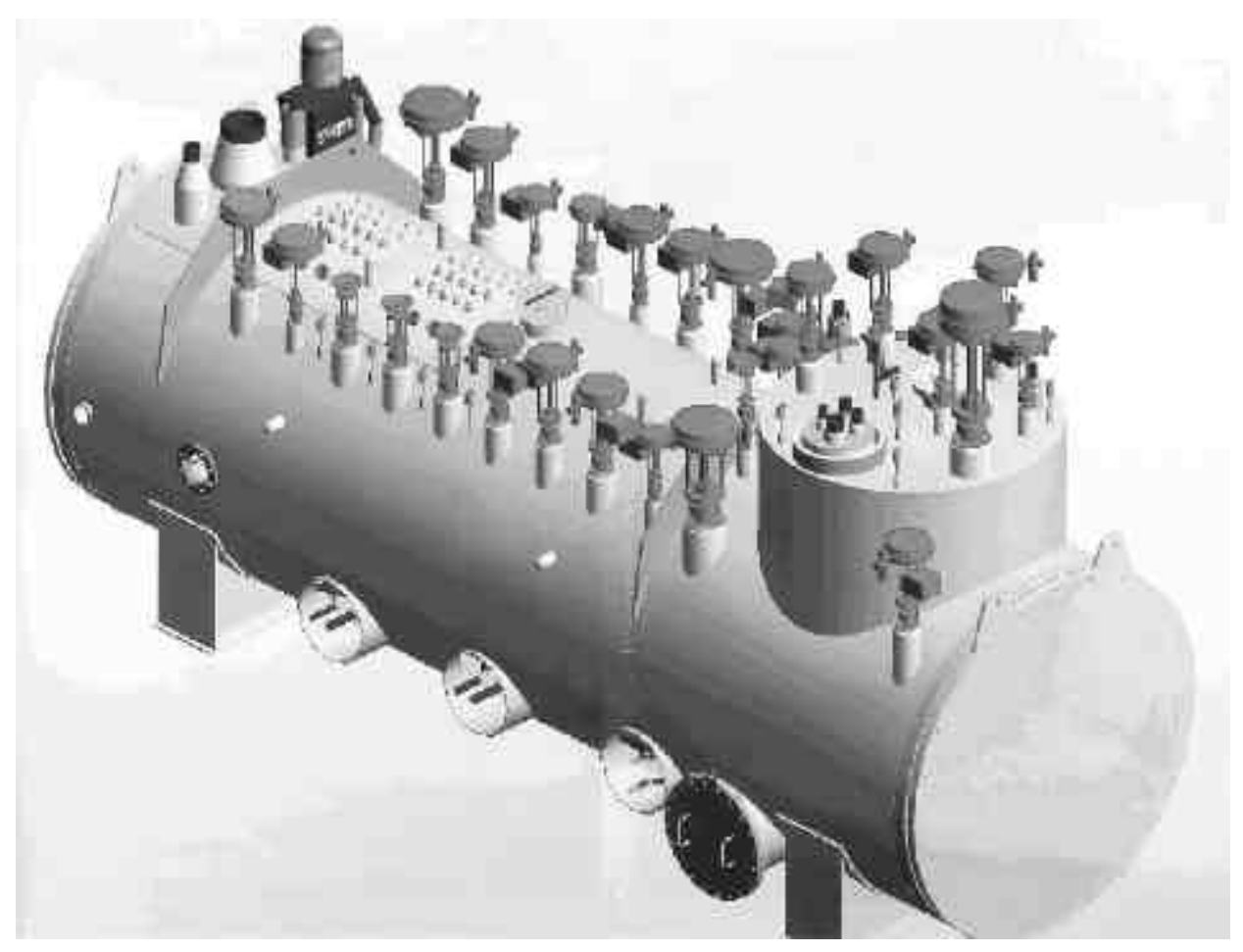

FIGURE 3. CAD view of the cold box.

the first and the second stage. The oil will be cooled from $84^{\circ} \mathrm{C}$ and $81^{\circ} \mathrm{C}$ to $50^{\circ} \mathrm{C}$. Cooling water is supplied at a temperature of $24^{\circ} \mathrm{C}$ and returned at $34^{\circ} \mathrm{C}$. There will be separate cooling water circuits for each compressor motor.

Two helium buffers, each of $250 \mathrm{~m}^{3}$ will be available for the pressure control of the compressor station and for the storage of the process helium during shut downs.

\section{Cold Box}

The cold box is $2 \mathrm{~m}$ in diameter and $5.9 \mathrm{~m}$ in length - dimensions which were imposed by the necessity to move the fully assembled cold box to its underground position after the surface tests. The cold box will be integrated by Air Liquide's own workshop. It includes one liquid nitrogen cooled precooler and five Marston ${ }^{\mathrm{TM}}$ plate fin heat exchangers grouped in three heat exchanger blocks. Furthermore the cold box will include one $80 \mathrm{~K}$ adsorber, one $20 \mathrm{~K}$ adsorber, one phase separator of $120 \mathrm{l}$ and, last but not least, three Air Liquide gas bearing turbines [3,4]. A CAD view of the cold box is shown in FIGURE 3.

Each of the 3 turbines is protected by its own dedicated safety PLC.

\section{Intermediate Cryostat}

In order to ensure an uninterrupted supply of liquid helium to the magnet thermosiphon cooling system for at least five hours and to also allow a slow discharge of the energy stored in the magnet $(2.6 \mathrm{GJ})$ in case of utility failure or cryoplant failure, the refrigeration system is equipped with a 60001 liquid helium cryostat called the intermediate cryostat. The intermediate cryostat is a vacuum-insulated dewar comprising a thermal shield, which is cooled at $80 \mathrm{~K}$ by the shield cooling circuit in series with the magnet shield. Both the internal vessel and the shield are equipped with 30 layers of superinsulation. 


\section{Test Cryostat}

The refrigeration system shall be thoroughly tested before it will be connected to the CMS magnet. Therefore, a test cryostat will be installed temporarily to simulate the loads and the functionality of the magnet during cool down, operation and failure cases.

The test cryostat comprises one circuit with two $20 \mathrm{~kW}$ heaters that will simulate the heat load of the magnet during cool down, one liquid helium reservoir of about 4001 equipped with a $1 \mathrm{~kW}$ heater to simulate the heat load in normal operation, one circuit with a $10 \mathrm{~kW}$ heater to simulate the current leads and, last but not least, one circuit with a heater of $7 \mathrm{~kW}$ to simulate the thermal load on the shields.

\section{Instrumentation}

All instrumentation for the CMS refrigeration system has been chosen considering CERN-wide standardisation for cryogenic plants.

The temperature measurement inside the cold box will be achieved by the means of silicone diode sensors. The sensors have been grouped by class of precision. The high precision sensors are in direct contact with the cryogen and can be replaced without breaking the cold box vacuum.

In most cases standard industrial components could be chosen for the instrumentation. The only exception being the intermediate cryostat that will be subject to a magnetic field of up to $200 \mathrm{G}$ and irradiation of about $2 \mathrm{~Gy}(10 y e a r s)^{-1}$, consisting mainly of neutron irradiation with $10^{11} \mathrm{~cm}^{-2}$ (10years) ${ }^{-1}$ neutrons at more than $100 \mathrm{keV}$. In spite of the low radiation levels compared with the ATLAS detector, the other LHC experiment requiring cryogenics, it has been agreed for standardisation reasons to apply the same principles for both the ATLAS and the CMS cryogenic systems. As consequence, no radiation sensitive electronics will be installed on or near the intermediate cryostat.

\section{Infrastructure}

The CMS refrigeration plant is connected to both the Swiss and the French highvoltage networks $(18 \mathrm{kV})$. In normal operation either network supplies one of the two compressors. In case of failure of one of the networks, the other network takes over the full load.

The cooling water consumption of the refrigeration plant will be $115 \mathrm{~m}^{3} / \mathrm{h}\left(24^{\circ} \mathrm{C}\right.$ to $34^{\circ} \mathrm{C}$ ). The cryogenic system is supplied via a dedicated circuit with redundant pumps from the common refrigeration tower for the CMS site.

The instrument air for the CMS refrigeration system components both on the surface and underground is supplied by a dedicated air compressor station. The instrument air system will be backed up by pressurised gaseous nitrogen supplied by an evaporator on the liquid nitrogen dewar.

\section{CONTROL SYSTEM}

The control system for the CMS refrigeration system is provided by the unified control system that has been adopted by CERN for all LHC cryogenic systems.

For the CMS refrigeration system a distributed control architecture comprising one PLC for the compressor station, one for the cold box, and one for the cryostat and the 


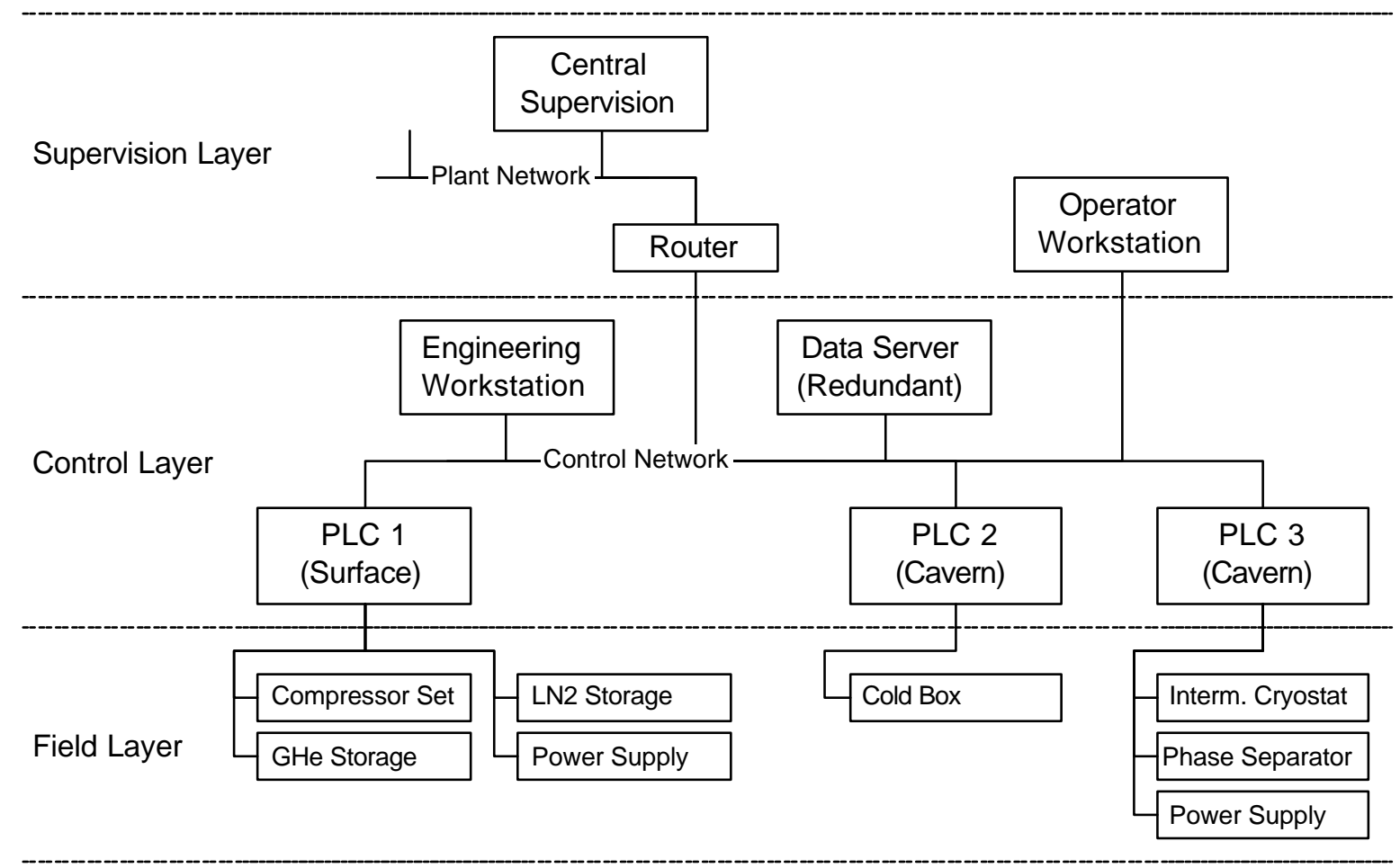

FIGURE 4. Controls architecture for the CMS refrigeration system.

phase separator controls has been chosen. It is composed of 3 Quantum $^{\text {TM }}$ PLCs type 534. The process shall be supervised by the MS Windows ${ }^{\mathrm{TM}}$ based control system PCVUE $32^{\mathrm{TM}}$. The controls architecture is shown in FIGURE 4.

\section{COMMISSIONING AND TESTS}

The commissioning of the refrigeration system with the test cryostat simulating the magnet is foreseen to start in June 2002. It is planned to extensively test all operation modes and failure modes of the refrigerator.

After connection to the phase separator and therewith to the thermosiphon cooling system, a first cool down of the magnet will allow mechanical and electrical tests on the fully assembled magnet system while on the surface and before its removal to the final underground position.

\section{CONCLUSION}

Air Liquide has designed and is constructing a helium refrigeration system as a turnkey project devoted to the cooling of the CMS particle detector at CERN.

Both the process cycle and the employed technologies have been chosen to meet the specific needs of the CMS superconducting magnet with high availability, reliability, and adaptability to various operating conditions. 


\section{ACKNOWLEDGMENTS}

The authors wish to thank their colleagues members of the "CMS project team" at Air Liquide and at CERN for their contributions to the design study of this refrigeration system and especially Y. Bonnet, Y. Cardet, T. Dupont, C. Guérin, C. Mantileri, G. Marot.

\section{REFERENCES}

1. Delikaris, D. et al., "The Cryogenic System for the Superconducting Solenoid Magnet of the CMS Experiment" in Proceedings of the 15th International Conference on Magnet Technology, Science Press, Beijing, pp.1230-1233.

2. CMS Collaboration, The compact muon solenoid, CERN/LHCC-94-38 LHCC/P-1, Geneva, 1994.

3. Marot, G. and Villard, J.C., "Recent Developments of Air Liquide Cryogenic Expanders" in Advances in Cryogenic Engineering 45B, edited by Quan-Sheng Shu et al., Plenum, New York, pp.1493-1500.

4. Villard, J.C., "Gas Bearing Expansion Turbines for Industry, Installation of the $300^{\text {th }}$ Machine Worldwide" in Cryogas International (Aug/Sept 1996). 